Case Report

iMedPub Journals

www.imedpub.com

DOI: $10.21767 / 2254-6758.100088$

\section{Surgical Treatment of Malignant Cerebral Infarction}

\section{Abstract}

Cerebrovascular disease (CVD) is the second cause of death in the world and the first in Uruguay. The malignant cerebrovascular accident (CVA) represents $5 \%$ of the cases of cerebral ischemia in our country. In these patients, the use of preoperative intracranial pressure monitoring has not shown utility. Currently, decompressive craniectomy has managed to reduce the mortality and morbidity of patients with malignant stroke. The clinical case of a patient who presented a malignant CVA is described below and a non-systematic review of the literature on the surgical treatment of them is performed.

Keywords: Stroke; Malignant infarction: Middle cerebral artery; Internal carotid artery infarction; Depressive hemicraniectomy

\section{Gonzalo Bertullo}

Department of Neurosurgery, Clinical Hospital "Dr. Manuel Quintelas", Faculty of Medicine, University of the Republic, Montevideo, Uruguay

\section{*Corresponding author: Gonzalo Bertullo \\ झ gonzalobertullo@hotmail.com}

Department of Neurosurgery, Clinical Hospital “Dr. Manuel Quintelas”, Faculty of Medicine, University of the Republic, Montevideo, Uruguay.

Tel: +59899516256

Citation: Bertullo G (2017) Surgical

Treatment of Malignant Cerebral Infarction. J Univer Surg. Vol.5 No.4:20
Received: November 17, 2017; Accepted: November 17, 2017; Published: November 17,2017

\section{Introduction}

CVD is the second cause of death in the world according to data from the World Health Organization (WHO) [1,2] Changes in the lifestyle and aging of the Uruguayan population have determined an increase in the prevalence of risk factors for the development of cardiovascular and cerebrovascular disease. In Uruguay, CVD was recognized as the leading cause of death in 2012, with 75.10 deaths due to this cause per 100,000 inhabitants [3].

CVD causes disabling sequelae creating a functional dependency and changes in the whole family structure. Malignant CVA are a pathology with significant mortality. In the following work we discuss the advances in the surgical treatment of malignant CVA from a clinical case.

\section{Case Report}

A 58-year-old male patient with a personal history of hypertension without treatment or control, smoker, and physical examination for morbid obesity. Five hours before the consultation install motor deficit of right hemicuerpo and alterations in the language. On admission examination, a vigil patient who fulfills orders with a left hemibody was observed. Aphasic mutism and right hemiplegia hemiplegia, presenting a total of 11 points on the Glasgow coma scale (GCS).

Cranial tomography (CT) showed incipient elements of ischemia in the territory of the middle cerebral artery (MCA) and left anterior cerebral artery (ACA) (Figure 1).

Intravenous fibrinolytics were not performed because they were out of time. At 17 hours after the onset of symptoms, the patient depresses consciousness, confirming eye opening to the call, does

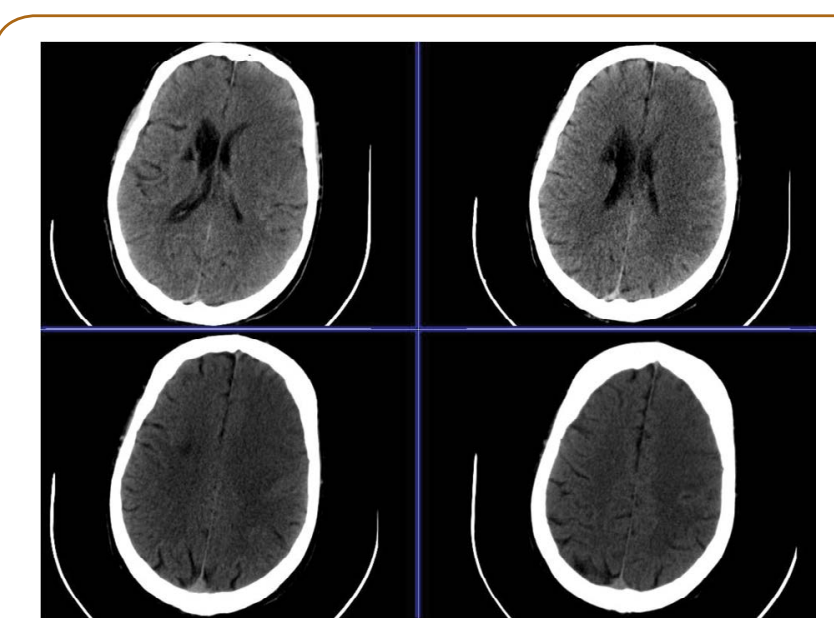

Figure 1 Cranial tomography at $5^{\text {th }}$ hour from the beginning of the Cerebrovascular Accident.There is a loss of differentiation between the gray and white matter associated with a hypodense area in the territory of anterior cerebral artery and left middle cerebral artery. 
not follow orders and persists with aphasic mutism, adding a GCS of 9. The CT scan showed a large hypodense area in superficial and deep territory of the silviana artery and the anterior cerebral artery, with a mass effect due to ipsilateral ventricle compression and a $4 \mathrm{~mm}$ mean line deviation (Figure 2). Given the clinical state of the patient and the tomographic findings, it was decided to perform a decompressive craniectomy. In the postoperative period, the patient is admitted to an intensive care unit (ICU) where he remains sedated and in mechanical ventilation. Upon examination, it does not present an eye opening to the call, nor gestures and locates with the left upper limb, GCS 7.

The intracranial pressure figures (ICP) were less than $20 \mathrm{mmHg}$ in the following 24 hours. The postoperative control CT showed an area of ischemia configured in the aforementioned territories and an increase in the mass effect with respect to the previous CT scan (Figure 3). At 72 hour after the onset of symptoms, ICP values reach $40 \mathrm{mmHg}$ and anisocoria is installed with left mydriasis. In this situation, the control CT evidences a configured infarction of the whole territory of the internal or panhemispheric carotid artery, an increase in the mass effect with a mean line deviation of $20 \mathrm{~mm}$, uncal herniation and subfalcin. The volume of the ischemia area was 700 cc (length $\times$ thickness $\times$ height $\times$ 0.63) (Figure 4).

The ecodoppler reported neck vessels, atheromatosis of the carotid bifurcation that extends to the internal carotid causing a subocclusive flow. A vigorous neurointensive medical treatment was performed, reversing the mydriasis. The patient dies on the 6th day of the CVA.

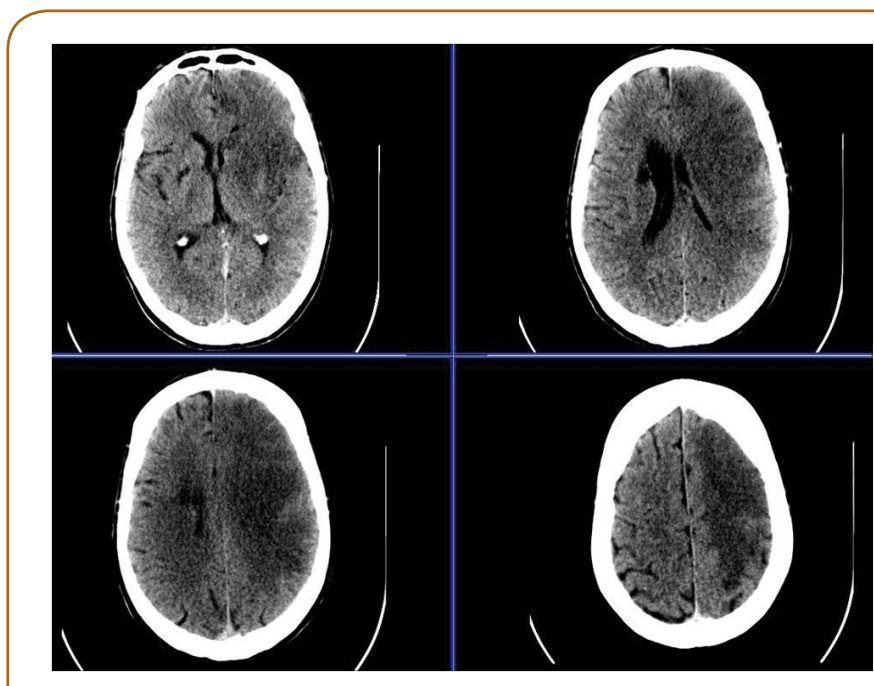

Figure 2 Cranial Tomography at $19^{\text {th }}$ hour of the Cerebrovascular Accident. Increase of the hypodense area with clear commitment of the superficial and deep territories of the Middle Cerebral Artery and Anterior Cerebral Artery to the left. Presents ipsilateral ventricle compression and deviation from the $4 \mathrm{~mm}$ midline.

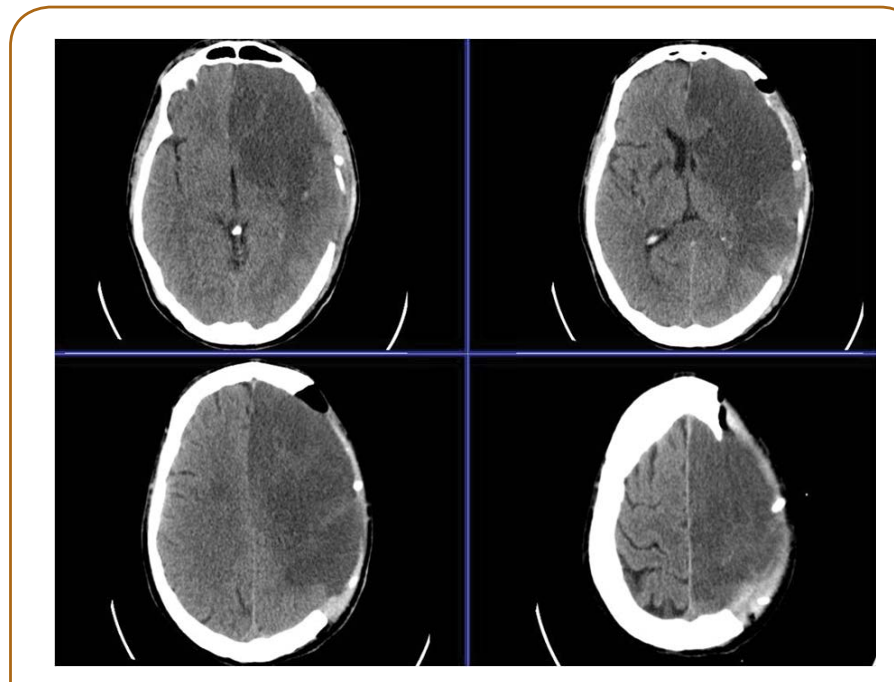

Figure 3 Cranial Tomography at $48^{\text {th }}$ hour after Cerebrovascular Accident and decompressive post-craniectomy. Ischemia configuration and increased mass effect are evidenced, with homolateral ventricle compression and deviation of the $6 \mathrm{~mm}$ midline.

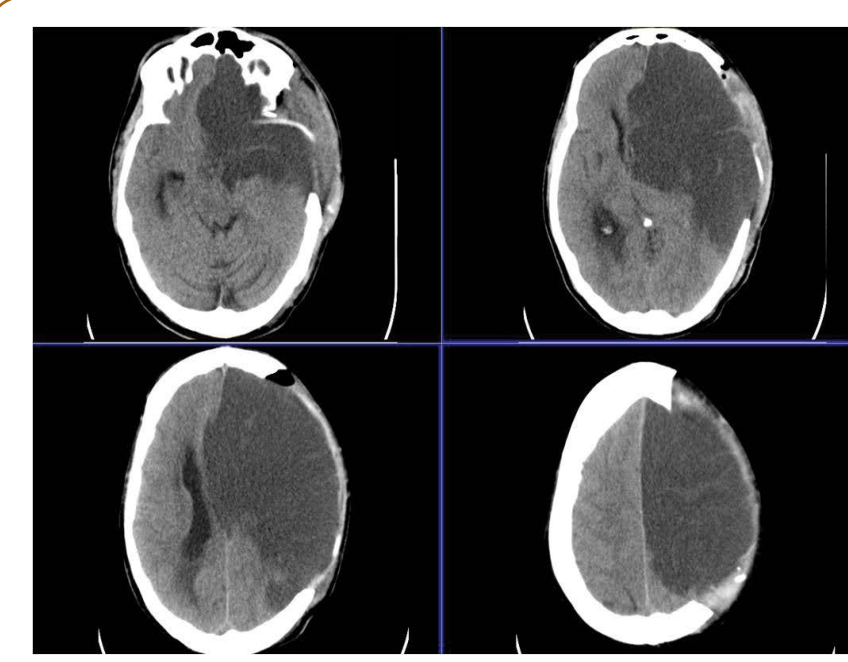

Figure 4 Cranial Tomography at $96^{\text {th }}$ hour of the Cerebrovascular Accident. The already configured pan hemispheric infarction is appreciated. Great increase in mass effect with ipsilateral ventricle compression, $20 \mathrm{~mm}$ midline deviation and contralateral hydrocephalus. Note the subclinical and uncal herniation with compression of the brainstem. The encephalic parenchyma protrudes through the hemicraniectomy.

\section{Discussion}

The term malignant CVA was first used by Hacke [4]. The term "malignant" arises as a result of the death of $78 \%$ of patients in the study, secondary to uncal and transtentorial herniation in 5 days after stroke, despite the optimal neurocritical treatment. In Uruguay, it is estimated that $5 \%$ of stroke is malignant, according to a study carried out in 2014 [5]. Currently, the term malignant CVA can be defined according to the inclusion criteria 
used in the only controlled clinical trials conducted up to the present time. According to these, we divide them based on: clinical, evolutionary and imaging criteria. The clinical criteria include the sudden installation of symptoms in the territory of the MCA. Symptoms linked to other cerebral vascular territories may also be included. The evolutionary criterion implies a decrease in the level of progressive consciousness from the onset of symptoms. This depression of consciousness is observed more frequently in the first 3 days, although it can be extended until the fifth day, period of time in which the increase in edema linked to ischemia is usually observed. However, although the value of the NIHS is variable, it is accepted as valid greater than 16. The imaging criteria consist in the appearance of signs that suggest an ischemia of at least $50 \%$ of the territory of the MCA, or a volume of the CVA greater than $145 \mathrm{cc}$ in the diffusion [6-12]. It is defined as a complete ACM infarction when there is a commitment of its three territories, anterior, posterior and deep; extensive when it commits at least $50 \%$ of it; and panhemispheric, when it also involves the MCA, ACA and / or the posterior cerebral artery (PCA) [13].

Our patient meets the clinical, evolutionary and imaging criteria to be included in the definition of malignant stroke. Clinically it debuted with sign-symptomatology suggestive of ischemia in the territory of the MCA, evolutionarily depressed consciousness passing from a GCS of 11 to 9 and imaging was a panhemispheric infarction (Figure 2). In the clinical case presented, we do not have a magnetic resonance imaging (MRI). The diffusion sequence in this patient could have provided information about the vascular territory involved from the beginning of the picture.The high mortality of these patients despite an excellent neurocritical treatment, has led to discuss the usefulness of surgical techniques with the general objective of improving the vital prognosis; and the specific and most important for many authors, of "saving brain function", allowing patients to be reintegrated into society with a better quality of life.

\section{Monitoring of Pic}

The monitoring of preoperative ICP in the CVA has not shown utility. It has been found that patients with a clear depression of consciousness and even with clinical and imaging elements of uncal herniation show no increase in ICP when it is monitored [14].

However, several authors emphasize the importance of ICP monitoring in the postoperative period. This not only allows us to predict the presence of complications after surgery, but also allows for adequate medical treatment in patients who, despite decompressive craniectomy, show an increase in ICP $[15,16]$.

In the case of our patient, he presented an episode of increased ICP with figures of $40 \mathrm{~mm} \mathrm{Hg}$, coinciding with a left mydriasis, which regresses with the treatment established. Subsequently, there were no new ICP elevations, which did not correlate with the severe mass effect observed on CT.

\section{Decompressive hemicraniectomy}

After obtaining the results of three controlled clinical trials in Europe, DESTINY, DECIMAL and HAMLET, the benefits of decompressive craniectomy have been demonstrated in the treatment of malignant CVA in patients under 60 years of age. In two meta-analyzes carried out that bring together the results of the three studies mentioned above, a reduction in mortality of $70 \%$ was shown in patients undergoing conservative treatment, to $20 \%$ in those in whom decompressive craniectomy was performed $[6,11]$. These works also showed a functional improvement in those patients treated surgically. The Modified Rankin Scale (mRS) is a scale designed to measure the degree of disability of neurological patients. 0 would be those asymptomatic individuals. 5 are patients confined to stay in bed. 6 are the deaths. It is considered that a scale between 0 and 3 or 4 is a good functional response.

It is verified that decompressive craniectomy increases $30 \%$ patients with mRS 4 and $10 \%$ patients with mRS 2 and 3 , however, there was no significant change in the percentage of patients with $\operatorname{mRS} 5[6,11]$.

These results are compelling. The possibility of presenting an $\mathrm{mRS}$ equal to or less than 4 was $75 \%$ for the treated group and $24 \%$ for the control group, with an absolute risk reduction of $51 \%$ (Cl: 34-69). This determines a necessary number of treat (NNT) of 2. That is, it is necessary to treat 2 patients with decompressive craniectomy so that one year at least one of them will be walking with help. To obtain an mRS less than or equal to 3 , an NNT of 4 is required and to reduce mortality, the NNT must be 2 [11]. Although there is currently acceptance of the benefit of decompressive craniectomy in patients under 60 years of age, some controversial points persist.

The first element to discuss is that it occurs with patients who present with a malignant cerebrovascular accident in the dominant hemisphere, as in the case of our patient. The three controlled clinical trials to which we have mentioned, show that there are no differences in terms of the functional evolution of patients based on the cerebral hemisphere involved. It has been found that symptoms, such as space heminegligence, can be as invalidating for patients as aphasia. Therefore, the evidence demonstrates the benefit of surgical treatment regardless of the cerebral hemisphere involved $[6,8,9,11,12]$.

The second controversial point is age. The DESTNY II was designed with the aim of assessing the difference in patients older than 61 years with conservative and surgical treatment. This study includes patients between 61 and 82 years. It showed a decrease in mortality of $30 \%$ in favor of patients in whom a decompressive craniectomy was performed. However, $60 \%$ of patients present with a mRS of 4 and 5, that is, they need help to satisfy their basic needs or they are confined to stay in bed. Only $7 \%$ of patients have a mRS of $3[10,17]$.

Inamasu et al. analyzed the results of surgical treatment in patients older than 61 years, separating patients into two groups, those between 61 and 70 years and those over 70 years. Although this study was carried out with a low number of patients, a mortality of $60 \%$ was found in the group of patients older than 70 years. There was no death in the group between 61 and 70 years [18]. These studies show that surgical treatment in patients 
between 61 and 70 years should be taken into account, analyzing each case in particular.

As a third controversial point, we must analyze which is the best opportunity for performing surgical treatment. There is no discussion that early treatment is the one that most benefits these patients. The HAMLET study allowed to know that, in the patients treated in the first 48 hours with surgery, there is a difference in mortality of $50 \%$, with respect to the conservative treatment. But in those operated after 48 hours, the improvement in mortality is less than $10 \%[6,8]$. However, these results are relative, since as we know the edema occurs between the day of the onset of symptoms and the fifth day. Those patients who present an initial depression of consciousness beyond the first 48 hours could also benefit from surgery.

Finally, the existing discussion on the increased risk of hemorrhage is highlighted in patients in whom intravenous or intra-arterial fibrinolytics were performed. Fibrinolytics promote the transformation of plasminogen into plasmin, thereby accelerating the degradation of the fibrin clot. The plasminogen recombinant tissue activator ( $r$-TPA), used in our country, has a half-life of $5 \mathrm{~min}$, however, it is considered that the fibrinolytic effect persists for more than 24 hours Three publications have been published in recent years that demonstrate that the performance of intravenous or intra-arterial fibrinolytics does not increase the risk of hemorrhagic complications in patients undergoing decompressive craniectomies, even when this is done in the first 24 hours after the administration of these drugs [19-21].

Despite the new evidence obtained, there is still a great lack of knowledge on the part of doctors of all specialties about the benefits of decompressive craniectomy. In a study conducted in Japan in 2014, it was found that less than $10 \%$ of patients with a malignant cerebrovascular accident had received surgical treatment. In more than $50 \%$ of the cases, it had been carried out late when elements of uncal herniation already existed $[5,22]$.

In the case presented, because it is a patient under 60 years of age, who is in the first 24 hours after the installation of the symptoms and with a GCS 9 to correct for aphasic mutism, the indication for surgical treatment is clearly justified. As we have analyzed, a stroke of the dominant hemisphere does not contraindicate the surgical treatment.

\section{Other surgical techniques}

Another surgical tactic mentioned in the literature is the resection of the ischemic cerebral parenchyma $[23,24]$. Kostov studied the benefit of infartectomy with respect to decompressive

\section{References}

1 Murray C, Lopez A (1997) Mortality by cause for eight regions of the world: Global Burden of Disease Study. Lancet 349: 1269-1276.

2 World Health Organization (2014) The 10 leading causes of death in the world, 2000 and 2012. Fact Sheet No. 310.

3 Arrarte GD, Lluberas R, Caorsi WR, Pereyra JJ, Draper R, et al. craniectomy. Although it is a work with a low number of patients, it shows that there is no significant change in mortality, but with a better prognosis in the functional of the patients in whom an infartectomy was performed. However, it tends to be more conservative in patients with strokes of the dominant hemisphere, performing decompressive craniectomies, which could have a translation in the functional differences observed [24]. Other authors state that the infartectomy could injure penumbra areas, negatively affecting the functional evolution of the patients.

We emphasize that some authors propose the realization of a temporal lobectomy with resection of the hippocampus uncus. With the aim of avoiding compression on the brainstem $[25,26]$. Undoubtedly new works are needed that show the possible benefits of these treatments.

\section{Prognostic factors}

The most important prognostic factors, in patients with a malignant cerebrovascular accident, who undergo surgical treatment, can be separated into clinical and imaging. The most recognized clinical criteria are age under 60 years, a relatively good GCS, the absence of pupillary changes and the time of evolution of symptoms. The most important imaging criterion as a predictor of mortality is the stroke volume greater than $200 \mathrm{cc}$. A deviation of the median line greater than $10 \mathrm{~mm}$ after decompressive craniectomy and the involvement of more than one vascular territory are also described [22, 27, 26, 28, 29].

In the case mentioned, the patient presents clinical elements, which translate a good prognosis. However, the presence of a CVA with a volume of $700 \mathrm{cc}$, three times the value considered as a limit to predict a poor prognosis, the deviation of the average line of $20 \mathrm{~mm}$ despite the decompressive craniectomy and the presence of a compromise of the ACA and MCA are all elements that significantly increase the probability of death of the patient [30].

\section{Conclusion}

The studies carried out in Europe in the last decade have made clear the benefit of decompressive craniectomy in the treatment of patients under 60 years of age with stroke, even with a commitment from the dominant hemisphere. However, for many neurosurgeons, treatment is still controversial in patients over 60 years of age, especially if we take into account the poor functional response shown by these patients. There are new lines of treatments under study that need greater scientific rigor before they can be recommended.

(2012) Honorary Commission for Cardiovascular Health. Area of Epidemiology and Statistics. Uruguay. Mortality due to cardiovascular diseases in Uruguay. Montevideo: CHSC : 1- 53.

4 Hacke W, Schwab S, Horn M, Spranger M, De Georgia M, et al. (1996) Malignant middle cerebral artery territory infarction: clinical course and prognostic signs. Arch Neurol 53: 309-315.

5 Brunet F, Camejo C, Gaye A, Castro L, Puppo C, et al. (2014) 
Cerebrovascular ischemic attack in Uruguay: comunication of the primeros 34 casos trombolizados in the Hospital of Clínicas. Rev Med Urug 30: 31-48.

6 Flechsenhar J, Woitzik J, Zweckberger K, Amiri H, Hacke W, et al. (2013) Hemicraniectomy in the management of space-occupying ischemic stroke. J Clin Neurosci 20: 6-12.

7 Hofmeijer J, Amelink G, Algra A, Gijn J, Macleod M, et al. (2006) Hemicraniectomy after middle cerebral artery infarction with lifethreatening Edema Trial (HAMLET). Protocol for a randomised controlled trial of decompressive surgery in space-occupying hemispheric infarction. Trials 7: 29- 35.

8 Hofmeijer J, Kappelle L, Algra A, Amelink G, Van Gijn J, et al. (2009) Surgical decompression for space-occupying cerebral infarction (the hemicraniectomy after middle cerebral artery infarction with lifethreatening edema trial [HAMLET]): a multicentre, open, randomized trial. Lancet Neurol 8: 326-333.

9 Juttler E, Schwab S, Schmiedek P, Unterberg A, Hennerici M (2007) Decompressive Surgery for the Treatment of Malignant Infarction of the Middle Cerebral Artery (DESTINY). A randomized, controllled trial. Stroke 38: 2518-2525.

10 Juttler E, Unterberg A, Woitzik J, Bosel J, Amiri H (2014) Hemicraniectomy in older patients with extensive middle cerebral artery stroke. N Engl J Med 370: 1091-1100.

11 Vahedi K, Hofmeijer J, Juettler E, Vicaurt E,George B (2007) Early decompressive surgery in malignant infarction of the middle cerebral artery: a pooled analysis of three randomised controlled trials. Lancet Neurol 6: 215-222.

12 Vahedi K, Vicaut E, Mateo J, Kurtz A, Orabi M (2007) SequentialDesign, Multicenter, Randomized, Controlled Trial of Early Decompressive Craniectomy in Malignant Middle Cerebral Artery Infarction (DECIMAL TRIAL). Stroke 38: 2506-2517.

13 Aiyagari V, Diringer M (2002) Management of large hemispheric strokes in the neurological intensive care. Neurologist 8: 152-162.

14 Poca M, Benejam B, Sahuquillo J, Riveiro M, Frascheri L, et al. (2010) Monitoring intracranial pressure in patients with malignant middle cerebral artery infarction: it is useful? J Neurosurg 112: 648-657.

15 Johnson R, Maartens N, Teddy P (2011) Decompressive craniectomy for malignant middle cerebral artery infarction: Evidence and Controversies. J Clin Neurosci 18: 1018-1022.

16 Paldor I, Rosenthal G, Cohen J, Leker R, Harnof S (2015) Intracranial pressure monitoring following decompressive hemicraniectomy for malignant cerebral infarction. J Clin Neurosci 22: 79-82.

17 Yao Y, Liu W, Yang X, Hu W, LI G (2005) Is decompressive craniectomy for malignant middle cerebral artery territory infarction of any benefit for elderly patients? Surg Neurol 64: 165-169.

18 Inamasu J, Kaito T, Watabe T, Ganaha T, Yamada Y, et al. (2013) Decompressive hemicraniectomy for malignant hemispheric stroke in the elderly: Comparison of outcomes between individuals $61-70$ and $>70$ years of age. J Stroke Cerebrovasc Dis 22: 1350-1354.

19 Fischer U, Taussky P, Gralla J, Arnold M, Brekenfeld C, et al. (2012) Decompressive craniectomy after intra-arterial thrombolysis: safety and outcome. J Neurol Neurosurg Psychiatry 82: 885-887.

20 Ozdemir O, Ozbek Z, Vural M, Durmaz R, Cosan E (2014) Early decompressive surgery after combined intra-venous thrombolysis and endovascular stroke treatment. Clin Neuro Neurosurg 122: 66-69.

21 Takeuchi S, Wada K, Nawashiro H, Arimoto H, Ohkawa H (2012) Decompressive craniectomy after intravenous tissue plasminogen activator administration for stroke. Clin Neurol Neurosurg 114: 1312-1315.

22 Chen CC, Cho DY, Tsai SC (2007) Outcome and prognostic factors of decompressive hemicraniectomy in malignant middle cerebral artery infarction. J Chin Med Assoc 70: 56-60.

23 Henderson G (2004) Management of Massive Cerebral Infarct. Curr Neurol Neurosci Rep 4: 497-504.

24 Kostov D, Singleton R, Panczykowski D, Kanaan H, Horowitz M (2012) Decompressive hemicraniectomy, strokectomy, or both in the treatment of malignant middle cerebral artery syndrome. World Neurosurg 79: 480-486.

25 Johnson R, Maartens N, Teddy P (2011) Technical aspects of decompressive craniectomy for malignant middle cerebral artery infarction. J Clin Neurosci 18: 1023-1027.

26 Pillai A, Menon S, Kumar S, Rajeev K, Kumar A (2007) Decompressive hemicraniectomy in malignant middle cerebral artery infarction: An analysis of long-term outcome and factors in patient selection. J Neurosurg 106: 59-65.

27 Gupta R, Connolly S, Mayer S, Elkind M (2004) Hemicraniectomy for massive middle cerebral artery territory infarction. A systematic review. Stroke 35: 539-543.

28 Tu P, Liu Z, Chuang C, Yang T, Wu C (2012) Postoperative midline shift as a secondary screening for the long term outcome of surgical decompression of malignant middle cerebral artery infarcts. J Clin Neurosci 19: 661-664.

29 Wartenberg KE (2012) Malignant middle cerebral artery infarction. Curr Opin Crit Care 18: 152-163.

30 Suyama K, Horie N, Hayashi K, Nagata I (2014) Nationwide survey of decompressive hemicraniectomy for malignant middle cerebral artery infarction in Japan. World 82: 1158-1163. 